\title{
Gap junctions are essential for murine primordial follicle assembly immediately before birth
}

\author{
Zhen Teng ${ }^{*}$, Chao Wang ${ }^{*}$, Yijing Wang, Kun Huang, Xi Xiang, Wanbao Niu, Lizhao Feng, \\ Lihua Zhao, Hao Yan and Hua Zhang \\ State Key Laboratory of Agro-Biotechnology, College of Biological Science, China Agricultural University, \\ Beijing 100193, China \\ Correspondence should be addressed to C Wang; Email: wangcam@cau.edu.cn \\ ${ }^{*}$ (Z Teng and C Wang contributed equally to this work)
}

\begin{abstract}
The reserve of primordial follicles determines the reproductive ability of the female mammal over its reproductive life. The primordial follicle is composed of two types of cells: oocytes and surrounding pre-granulosa cells. However, the underlying mechanism regulating primordial follicle assembly is largely undefined. In this study, we found that gap junction communication (GJC) established between the ovarian cells in the perinatal mouse ovary may be involved in the process. First, gap junction structures between the oocyte and surrounding pre-granulosa cells appear at about $19.0 \mathrm{dpc}$ (days post coitum). As many as 12 gap junction-related genes are upregulated at birth, implying that a complex communication may exist between ovarian cells, because specifically silencing the genes of individual gap junction proteins, such as Gja1, Gja4 or both, has no influence on primordial follicle assembly. On the other hand, non-specific blockers of GJC, such as carbenoxolone (CBX) and 18 $\alpha$-glycyrrhetinic acid (AGA), significantly inhibit mouse primordial follicle assembly. We proved that the temporal window for establishment of GJC in the fetal ovary is from 19.5 dpc to 1 dpp (days postpartum). In addition, the expression of ovarian somatic cell (OSC)-specific genes, such as Notch2, FoxI2 and Irx3, was negatively affected by GJC blockers, whereas oocyte-related genes, such as Ybx2, Nobox and Sohlh1, were hardly affected, implying that the establishment of GJC during this period may be more important to OSCs than to oocytes. In summary, our results indicated that GJC involves in the mouse primordial follicle assembly process at a specific temporal window that needs Notch signaling cross-talking.

Reproduction (2016) 151 105-115
\end{abstract}

\section{Introduction}

The primordial follicle is the initial developmental stage of a follicle. The reserve of primordial follicles in the ovary is referred to as the primordial follicle pool. As the only source of mature oocytes, the primordial follicle pool determines the reproductive life of a female mammal (Burgoyne \& Baker 1981, Zhang et al. 2012). However, the mechanism leading to a successful establishment of a primordial follicle pool is still unclear (Smith et al. 2014).

A primordial follicle consists of two different types of cells: an oocyte in the center and the granulosa cells around it. Prior to primordial follicle formation, the incompletely divided oocytes aggregate in the form of syncytia, namely cysts (Bukovsky et al. 2005). The structure of cysts is preserved until parturition, when selective apoptosis of oocytes occurs, breakdown of cysts begins and individual oocytes are isolated (Pepling \& Spradling 2001, Wen et al. 2009). Simultaneously, ovarian somatic cells (OSCs) undergo programmed differentiation. For instance, the expression of forkhead box L2 (Fox/2), a marker of granulosa cells, indicates the committed fate of OSCs to become granulosa cells (Mork et al. 2012). Meanwhile, a temporal and quantitative synchronous development of oocytes and granulosa cells has been proved to be important for normal folliculogenesis (Lei et al. 2006, Li et al. 2011). However, the coordinator in this procession has not been well studied.

Gap junction communication (GJC) is a special cellular communication that allows direct substance exchange between cells through gap junction channels. In mice, as many as 20 gap junction-related genes, also termed connexins, participate in forming the channels. The channels allow passive diffusion of hydrophilic molecules smaller than $1 \mathrm{kDa}$, including second messengers, small metabolites and microRNAs (Kumar \& Gilula 1996, Valiunas et al. 2005, Wolvetang et al. 2007). Meanwhile, the selective permeability of 
connexins to these substances differs between distinct isoforms (Ek-Vitorin \& Burt 2013). Therefore, the specific temporal and spatial distribution of distinct connexins may contribute to the tissue-specific regulation of a wide range of physiological and cellular processes, including cell differentiation, proliferation and death (Lo 1999).

In the ovary, it has been well established that the regulating signals of meiotic maturation pass through the oocyte-granulosa cell gap junctions (Fagbohun \& Downs 1991, Downs 1995). Among these, gap junction proteins alpha 1 (Gja1, Cx43) and alpha 4 (Gja4, Cx37) are the most important gap junction genes in the mature ovary. Ovaries lacking Gja1 do not proceed beyond the primary follicle stage (Juneja et al. 1999, Ackert et al. 2001). In Gja4-deficient mice, folliculogenesis is arrested at the early antral stage (Simon et al. 1997). In addition, gap junction proteins, such as Gja10 (Cx57), Gjb1 (Cx32), Gjb2 (Cx26), Gjb3 (Cx31) and Gjc1 $(C \times 45)$, are detectable in the mature or $17.5 \mathrm{dpc}$ ovary (Kidder \& Mhawi 2002, Juneja 2003). Although none of them has a proven relationship with primordial follicle formation, previous work in our laboratory showed that non-specific blocking of GJC in the fetal ovary is able to suppress primordial folliculogenesis (Wen et al. 2009). These facts imply that the understanding of intercellular communication in the process of primordial follicle assembly is meaningful.

In this study, the role of gap junction during the formation of primordial follicles in the perinatal mouse ovary was studied. The results indicated that gap junction participated in the formation of the primordial follicle in a temporal window near birth, and may direct OSC differentiation during primordial follicle assembly.

\section{Materials and methods} Animals

CD1 mice were purchased from the Laboratory Animal Center of the Institute of Genetics in Beijing and kept in mouse facilities that met the requirements of the China Agricultural University Institutional Animal Care and Use Committee. Mice were housed in the China Agricultural University with a ratio of $16 \mathrm{~h}$ light: $8 \mathrm{~h}$ darkness at $26^{\circ} \mathrm{C}$, with free access to food (Rat \& Mouse Maintenance Diet 1022; HFK Bio-tech, China) and water. Female mice of 6-8 weeks old were mated with adult male mice. The presence of a vaginal plug was confirmed at 0.5 days post coitum (dpc) of pregnancy. The day of parturition was considered to be 0.5 days postpartum (dpp). All animal work was conducted using protocols approved by the China Agricultural University Institutional Animal Care and Use Committee (License No. SKLAB2014-03-01).

\section{Chemicals}

Unless otherwise noted, all chemicals were purchased from Sigma-Aldrich.

\section{Ovarian tissue dissection and in vitro culture}

Pregnant mice or pups were killed by cervical dislocation on the designated day. For in vitro culture of ovaries, fetal ovaries were dissected with micro-tissue forceps and syringe needle in cold sterilized PBS under a stereoscopic microscope (ZSA302; COIC, Chongqing, China) under sterile conditions. Ovaries were cultured in 24-well culture plates (NEST, Jiangsu, China) in $250 \mu \mathrm{l}$ pre-equilibrated DMEM/Ham's F12 nutrient mixture (DMEM/F12; GIBCO, Life Technologies) at $37^{\circ} \mathrm{C}, 5 \% \mathrm{CO}_{2}$ and saturated humidity. Half of the culture medium was replaced every 2 days until the ovaries had grown to the required stage.

Carbenoxolone (CBX, C4790) and 18a-glycyrrhetinic acid (AGA, G8503) are two gap junction blockers that work through different mechanisms. CBX inhibits the connection of two connexons, the hemichannel, while AGA inhibits the polymerization of gap junction protein monomers into connexons (Davidson et al. 1986, Krysko et al. 2004). These two treatment groups were used to exclude possible non-specific effects on ovary development. A stock solution of CBX $(20 \mathrm{mM})$ was prepared in PBS and then added to the medium to a working concentration of $20 \mu \mathrm{M}$. A stock solution of AGA $(10 \mathrm{mM})$ was prepared in DMSO and then added to the medium to $10 \mu \mathrm{M}$. In addition, the medium containing the same amount of DMSO $(1 \%, v / v)$ was used as a vehicle control group.

The bromodeoxyuridine (BrdU) incorporation assay was used for detecting ovarian cellular proliferation. BrdU (B5002) was added to the culture system to $10 \mu \mathrm{M}$ and incubated for $2 \mathrm{~h}$. The samples were then collected, washed and prepared for histological examination by immunohistochemical (IHC) assays.

\section{Kidney capsule transplantation}

All $17.5 \mathrm{dpc}$ fetal ovaries were cultured in either normal or CBX-containing medium for 7 days. These tissues were used for transplantation. Healthy adult female mice weighing about $20 \mathrm{~g}$ were chosen as recipients. Anesthesia was induced by intraperitoneal injection of $1 \%$ of animal weight, about $0.2 \mathrm{ml}$, pentobarbital sodium solution (P3761, 3\%, w/v in saline). Before transplantation, the recipient's ovaries were removed. Then, three control ovaries were transplanted to the subcapsular region of one kidney and three CBX-treated ovaries to the other kidney. After 2 weeks of culture in the kidney capsule, the grafts were collected for histological examination.

\section{Knock-down of Gja1 and Gja4 gene expression in fetal ovaries by RNAi}

The information of dsRNAs targeting Gja1 and Gja4 is given in Table S2, see section on supplementary data given at the end of this article. Moreover, for RNA interference with Gja1 and Gja4 simultaneously, the two specific dsRNAs were mixed in equal amounts. Before RNAi, $0.5 \mu$ of $20 \mu \mathrm{M}$ dsRNAs (Genepharma, Shanghai, China) were injected into $17.5 \mathrm{dpc}$ ovaries with glass pipettes under a stereoscopic microscope. Afterwards, ovaries were placed in chambers of the electric transfection apparatus (ECM2001; BTX, San Diego, CA, USA) to assist siRNA transfection. The parameters of the electric transfection were 
three 5 ms-long quasi-square pulses at a pulse-field strength of up to $40 \mathrm{~V} / \mathrm{cm}$. Total mRNA was then extracted to determine the efficiency of RNAi after 2 days of in vitro culture at $37^{\circ} \mathrm{C}$, $5 \% \mathrm{CO}_{2}$ and saturated humidity. The non-targeting siRNA sequence was 5'-ACGTGACACGTTCGGAGAATT-3', which is not homologous to any known mouse mRNA.

\section{Immunohistochemical and immunofluorescence staining}

All prepared samples for detecting DDX4, MKI67 or incorporated BrdU within the ovaries were fixed in 4\% PFA, dehydrated in an ethanol series, cleared in xylene, embedded in paraffin and sliced into $5 \mu \mathrm{m}$-thick sections. Sections on the slides were dewaxed in xylene and rehydrated in an ethanol gradient. Antigen retrieval was accomplished by boiling the sections in $0.01 \%$ sodium citrate buffer.

Antibodies were prepared as follows: for IHC staining, DDX4 (mouse, ab4300, 1:400; Abcam) primary antibody or BrdU (mouse, NBP2-29414, 1:400; Imgenex, CO, USA) primary antibody was diluted in PBS; for immunofluorescence (IF) staining, DDX4 (1:400) and MKI67 (rabbit, 9129S, 1:400; CST, MA, USA) primary antibodies were diluted in antibody dilution buffer (ADB) (3\% BSA, 1\% normal donkey serum in
Tris-buffered saline) as the primary antibody cocktail. Slides were then incubated with the primary antibody solution overnight at $4{ }^{\circ} \mathrm{C}$.

For IHC assay, slides were processed using the biotinstreptavidin HRP detection system (SP-9002; ZSGB-BIO, Beijing, China) followed by 3,3'-diaminobenzidine (DAB) staining, with counter-staining with hematoxylin. The slides were coverslipped with neutral resins for subsequent analysis. For IF assay, Alexa Fluor 555-conjugated Affinipure donkey anti-mouse (1:200, A-21202; Life Technologies) and 488conjugated donkey anti-rabbit secondary antibody (1:200, A-21432, Life Technologies) were applied to the slides, and then incubated for $2 \mathrm{~h}$ at $37^{\circ} \mathrm{C}$. The slides were rinsed in PBS and coverslipped with anti-fade fluorescence mounting medium (Applygen, Beijing, China) with Hoechst. The detection of DDX4 and MKI67 proteins on the slides of the ovaries was performed under a fluorescence microscope (Eclipse, Nikon, Japan).

To clarify the expression pattern of MKI67 in situ, each set of tissue sections from either treatment group was processed in one batch for IF assay to make the treatment conditions of each slide almost the same. Afterwards, to obtain a set of comparable results, exposure time for each signal, Hoechst (blue) and Alexa Fluor 488 (green) for MKI67 and Alexa Fluor

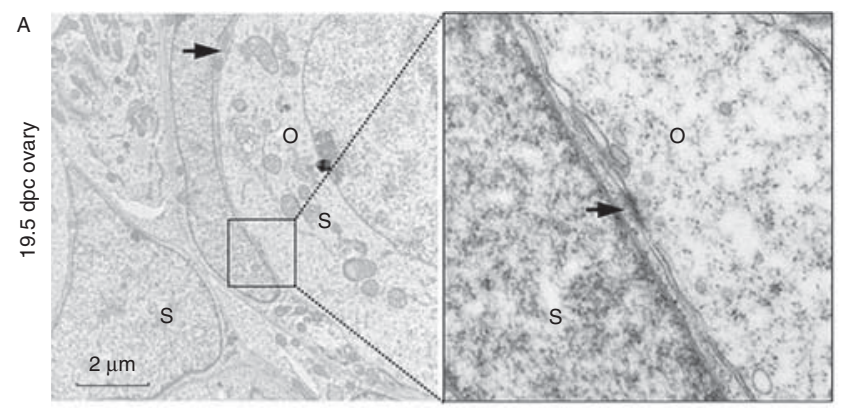

B

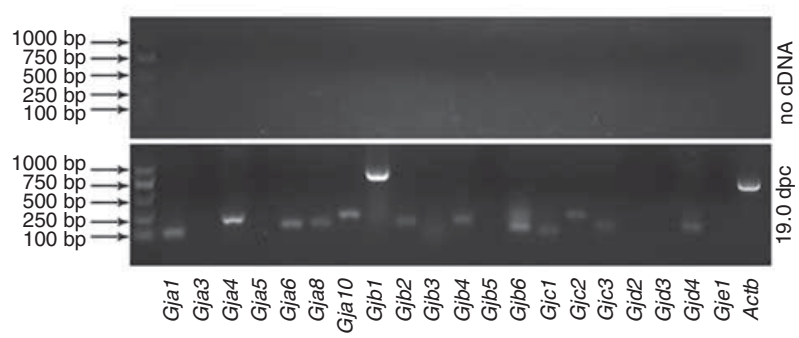

C

Group 1

Group 2
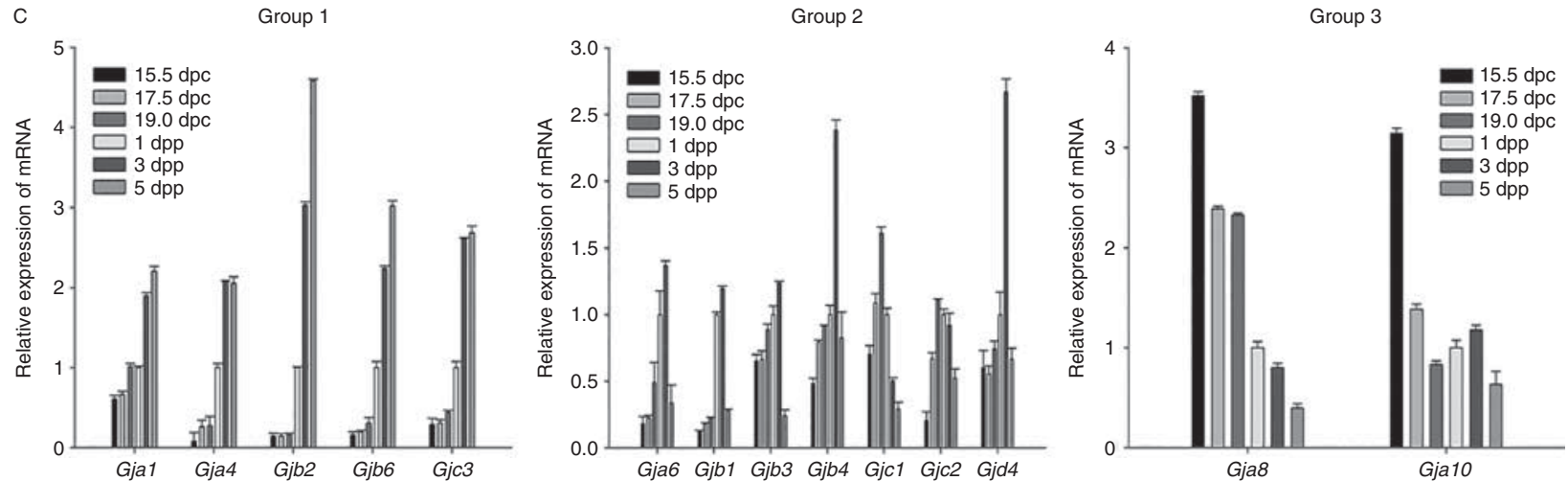

Figure 1 Existence of gap junctions in the fetal mouse ovary. (A) Transmission electron microscopy (TEM) of the gap junction structure in a 19.5 dpc ovary. Left: a TEM image of the 19.5 dpc ovary. ' $\mathrm{O}$ ' indicates oocyte; 'S' indicates ovarian somatic cells (OSCs). The arrow indicates typical highelectron density area of the gap junction structure. Scale bar: $2 \mu \mathrm{m}$. Right: a detailed view of the left, showing one of the gap junction structures observed in the 19.5 dpc ovary. This image is one representative section of three examined ovaries. (B) Electrophoresis result of RT-PCR amplification for gap junction genes in 19.0 dpc ovary cDNA. This result shows that 14 gap junction genes were expressed in 19.0 dpc ovary cDNA. Amplification without cDNA indicates that there were no non-specific amplification products for each primer. (C) Different expression patterns of gap junction genes. This qRT-PCR result shows three types of mRNA expression pattern of gap junction genes from $15.5 \mathrm{dpc}$ to $5 \mathrm{dpp}$ in the ovary. Group 1 shows genes that continuously increased; group 2 shows genes that decreased after birth; group 3 shows genes that continuously decreased. This is a representative result of the experiments that were repeated three times independently. 
555 (red) for DDX4, on each slide was also respectively the same. In detail, we exposed each sample to Hoechst for $50 \mathrm{~ms}$, Alexa Fluor 488 for 200 ms and Alexa Fluor 555 for 200 ms.

\section{Oocyte counting}

In this study, every three to five ovaries were randomly allocated into the control or treated group of one experiment. Cultured ovaries were fixed as described above and sliced into $5 \mu \mathrm{m}$ thick sections. Generally, a cultured ovary could be sliced into about 50 sections. After being immunostained with DDX4 antibody, every five sections were counted, considering that the diameter of a follicle is about $20-25 \mu \mathrm{m}$, which means that 10-12 sections in one ovary were counted. Numbers of oocytes, primordial follicles and primary follicles of each section were summed up respectively and multiplied by five to represent each index in total for one ovary. Mean and error of the three to five ovaries in each group were used to make a column in a chart.

\section{Quantitative real-time RT-PCR}

RNA was extracted using TRIzol (Invitrogen, Life Technologies) from five ovaries per sample according to the manufacturer's protocol, and $2 \mu \mathrm{g}$ total RNA were used for reverse transcription (Promega Reverse Transcription System).

For detecting the existence of gap junction members in the $19.0 \mathrm{dpc}$ ovary, we amplified the first-strand cDNA samples (10 ng) by using oligonucleotide primer pairs $(10 \mathrm{nM})$ in a PCR mix solution (ZT201A; Zoman Biotech., Beijing, China). Amplification consisted of a pre-incubation step at $94{ }^{\circ} \mathrm{C}$ for $5 \mathrm{~min}$ and then 45 cycles at $94{ }^{\circ} \mathrm{C}$ for $30 \mathrm{~s}, 60^{\circ} \mathrm{C}$ or $54{ }^{\circ} \mathrm{C}$ (for Gje1) for $30 \mathrm{~s}$, and $72{ }^{\circ} \mathrm{C}$ for $30 \mathrm{~s}$. Actin, (Actb) was used as the internal control. PCR using the same procedure but without cDNA samples was carried out for primer quality control. The information for primers of gap junction genes is given in Table S1, see section on supplementary data given at the end of this article.

Quantitative RT-PCR was performed and analyzed by using the Applied Biosystems 7300 Real Time PCR System (Life Technologies). Data were normalized by Gapdh. Primers for the genes tested are listed in Table S2, see section on supplementary data given at the end of this article.

\section{Transmission electron microscopy (TEM)}

Collected ovaries were fixed in $4 \%$ formaldehyde and $1 \%$ glutaraldehyde in $0.1 \mathrm{M}$ phosphate buffer (pH 7.4), washed in 8\% $(0.2 \mathrm{M})$ sucrose and post-fixed in $1 \%$ osmium tetroxide. Then, they were dehydrated in gradient ethanol solutions, propylene oxide and propylene oxide/EMBed 812 mixture. Tissues were embedded in EMBed 812 for sectioning. Tissues were cut into ultrathin sections $(80 \mathrm{~nm})$ and the sections were collected onto grids. The sections were then dried overnight for uranyl acetate and lead citrate staining. The stained samples were observed under an electron microscope (Tecnai G2 F30; FEl, Hillsboro, OR, USA). To analyze the existence of gap junction structure in ovaries, five to seven sections of each 19.5 or $17.5 \mathrm{dpc}$ fetal mouse ovary were examined. Most of these sections were located around at the position at 1/5-1/4 long axis of ovaries where there were abundant oocytes and diverse cell types.

\section{Statistical analysis}

All cultures, immunochemical and IF localizations were repeated at least five times using ovaries from different fetuses for each assay. Data are presented as means \pm s.E.M. with each model performed in triplicate or quintuplicate. Data were analyzed either by $t$-test or ANOVA. If a significant $F$ ratio was confirmed by ANOVA, data of the related groups were analyzed by the Holm-Sidak test. $P$ values $<0.05$ were considered statistically significant.

\section{Results}

\section{Expression pattern of connexins in the neonatal mouse ovary}

To investigate the existence of gap junctions in the mouse fetal ovary, transmission electron microscopy was
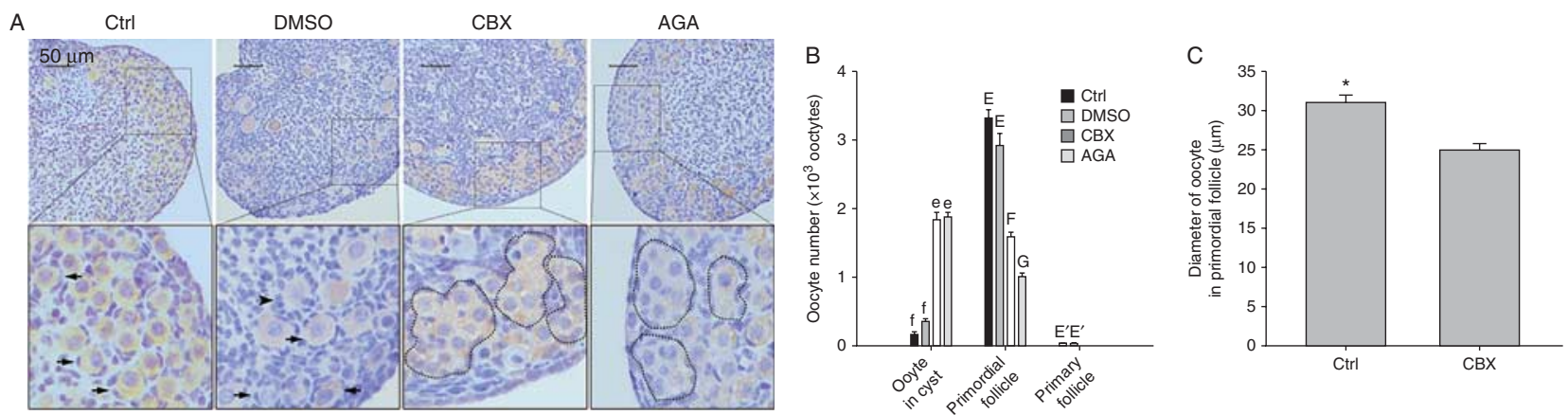

Figure 2 Effect of blocking gap junction formation on follicle assembly in the in vitro cultured fetal ovary. (A) IHC examination of $17.5 \mathrm{dpc}$ fetal ovaries treated with different gap junction blockers after 7 days of culture. The upper row presents an overall view of cultured tissues in the control $(\mathrm{Ctrl})$, vehicle control (DMSO), CBX- and AGA-treated groups. The lower row shows a detailed view of the same slice. DDX4 was immunostained (reddish brown) to label the oocytes. The structure of a typical cyst is encircled by the dotted lines; the arrow indicates typical primordial follicle; the arrowhead indicates typical primary follicle. Scale bar: $50 \mu \mathrm{m}$. (B) Developmental stage statistics of oocytes. In the CBX-and AGA-treated groups, primary follicles were not observed. The letters indicate significant differences between groups $(P<0.05 ; A N O V A)$. Data are presented as means \pm S.E.M. (C) Statistical analysis of the diameter of oocytes in primordial follicles. The asterisk indicates significant difference between the control and CBX-treated groups $(P<0.05 ; t$-test). Data are presented as means \pm S.E.M. All the experiments were repeated seven times independently. 
used to detect their typical high electron-dense linear area between opposing cell surfaces. As a result, the gap junction structure was evident in $19.5 \mathrm{dpc}$ ovaries (Fig. 1A), but seldom in $17.5 \mathrm{dpc}$ ovaries (Fig. S1, see section on supplementary data given at the end of this article). Meanwhile, in $19.5 \mathrm{dpc}$ ovaries, the gap junction structure was seen at the contact surface between the oocytes and OSCs, but hardly appeared at the sites between the oocytes themselves.

To determine how many gap junction genes were expressed in the fetal ovary, we used RT-PCR to examine the expression of all 20 mouse gap junction genes in the $19.0 \mathrm{dpc}$ ovary. As a result, Gja1, Gja4, Gja6, Gja8, Gja10, Gjb1, Gjb2, Gjb3, Gjb4, Gjb6, Gjc1, Gjc2, Gjc3 and Gjd4 were detectable at this time point (Fig. 1B). Furthermore, we used qRT-PCR to measure the relative expression changes of these gap junction genes in cDNA samples from ovaries at $15.5 \mathrm{dpc}, 17.5 \mathrm{dpc}, 19.0 \mathrm{dpc}, 1 \mathrm{dpp}, 3 \mathrm{dpp}$ and $5 \mathrm{dpp}$. As shown in Fig. 1C, there were three types of expression patterns of these gap junction genes. Gja1, Gja4, Gjb2, Gjb6 and Gjc3 comprised the first group, in which genes continuously increased with ovary development. The second group consisted of Gja6, Gjb1, Gjb3, Gjb4, Gjc1, Gjc2 and Gjd4. They were upregulated around $17.5 \mathrm{dpc}$, and their expression peaked at about $1 \mathrm{dpp}$ and then decreased at $5 \mathrm{dpp}$. Besides these, Gja8 and Gja10 were the only two genes that decreased in expression with ongoing fetal ovary development (Fig. 1C).

\section{Gap junction blockade inhibits cyst breakdown}

To investigate the gap junction function during primordial follicle formation, we chose two non-specific gap junction blockers, carbenoxolone $(\mathrm{CBX}, 20 \mu \mathrm{M})$

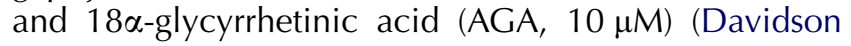
et al. 1986, Krysko et al. 2004) to treat in vitro cultured ovaries. This was based on two considerations. First, there are abundant gap junction genes existing in the fetal mouse ovary, and hence it was necessary to use non-specific blockers to eliminate all possible gap junction communication in the perinatal mouse ovary. Second, the use of two chemical compounds would help to exclude a possible non-specific effect.

After 7 days of culture from $17.5 \mathrm{dpc}$, ovaries developed to a stage equal to $5 \mathrm{dpp}$ when the primordial follicle pool should have been well established. In the control and vehicle ( $0.1 \%$ DMSO) groups, more than $80 \%$ of oocytes took the form of the primordial follicle, while the CBX- or AGA-treated ovaries had significantly fewer primordial follicles. On the other hand, in the CBX- and AGA-treated groups, there was a prominence of cysts, as revealed by continuous oocyte-specific cytoplasmic DDX4 staining (Pepling \& Spradling 2001). There were $53.6 \%$ (CBX-treated group) and $65.0 \%$ (AGA-treated group) of oocytes in the cysts, which were significantly higher than that in the control $(4.5 \%)$ and vehicle $(10.5 \%)$ groups $(P<0.05)$. Besides, the growth of follicles was also affected. The diameter of primordial follicles was smaller in the CBX-treated group when compared with the control $(25.24 \pm 0.89$ vs $31.38 \pm 0.88 \mu \mathrm{m}$; Fig. $2 \mathrm{C}$ ), and primary follicles, which developed in the control and vehicle groups, were absent in both treatment groups (Fig. 2A and B). In addition, the total oocyte numbers in all these groups were not significantly changed.
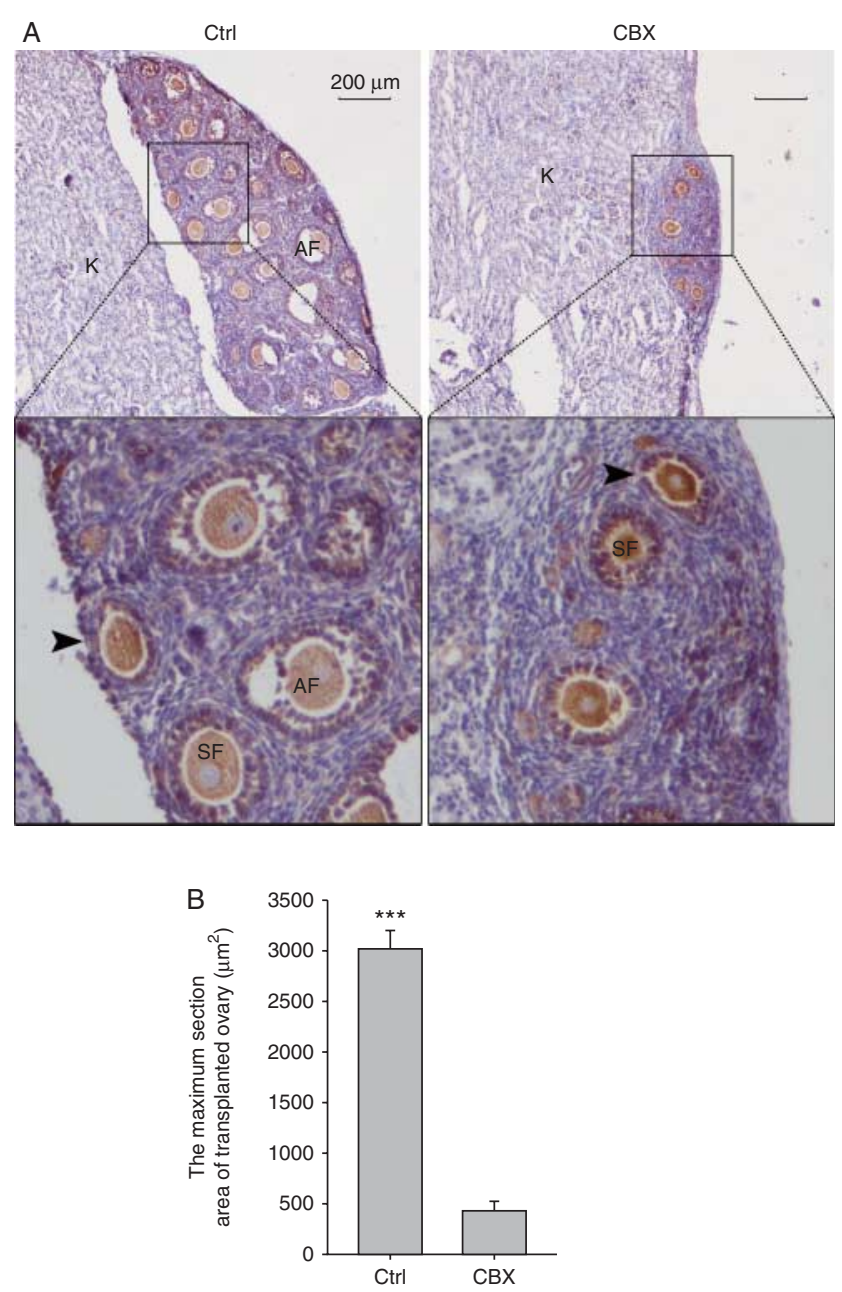

Figure 3 Effect of gap junction blockade during the perinatal period on long-term ovary development. (A) IHC examination of CBX-treated or control ovaries after 2 weeks of culture in the kidney capsule. The upper row presents an overall view of grafts in the control (Ctrl) and CBXtreated groups. The lower row shows a detailed view of the same slice. DDX4 was immunostained (reddish brown) to label the oocytes. The arrowhead indicates typical primary follicle; 'SF' indicates typical secondary follicle; 'AF' indicates typical antral follicle; ' $\mathrm{K}$ ' indicates kidney tissue. Scale bar: $200 \mu \mathrm{m}$. This experiment was repeated five times independently. (B) The statistics of the maximum section area of the transplanted ovary. The asterisk indicates significant difference between the control and CBX-treated groups $(P<0.001 ; t$-test). Data are presented as means \pm S.E.M. This experiment was repeated three times independently. 


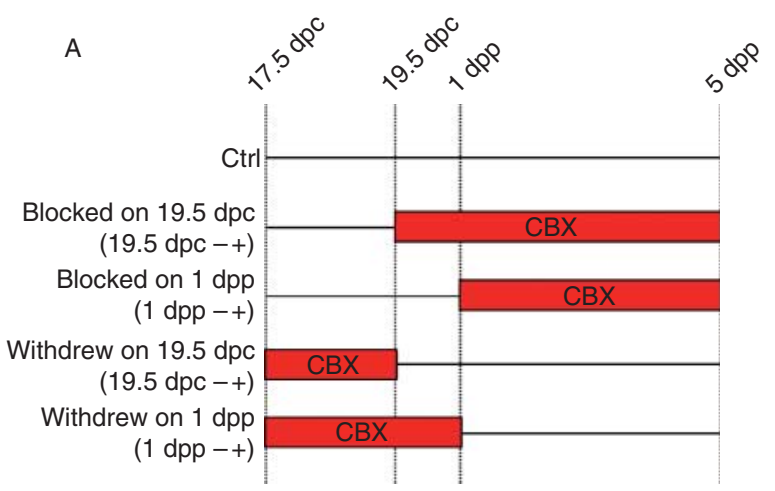

\section{B \\ Temporal window for gap junction function circling the perinatal period}
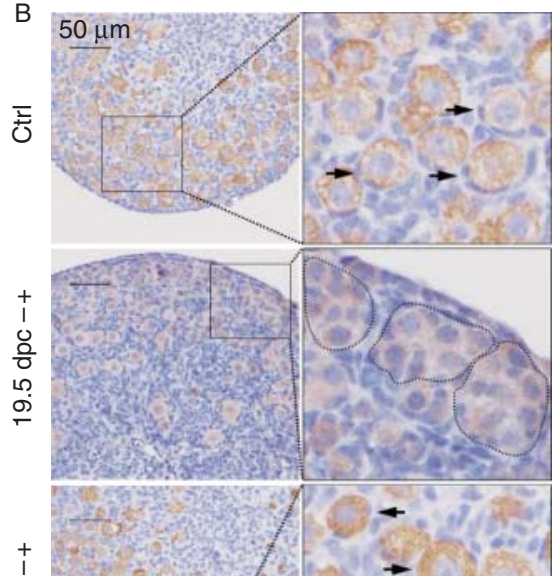

응
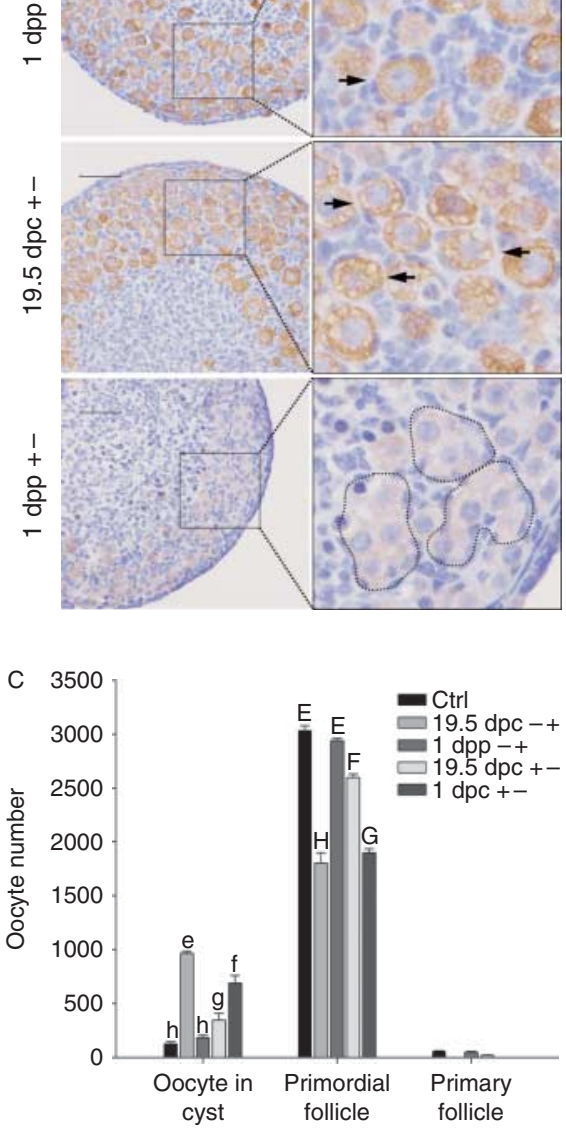

To investigate the long-term effect of GJC blockade, 7-day in vitro cultured ovaries were transplanted beneath the kidney capsule of ovariectomized adult female mice and cultured for 2 weeks. The control and CBX-treated ovaries were respectively transplanted into the capsules of opposite kidneys of the same mice. After 2 weeks of culture, many antral follicles developed in control ovaries, but the most developed follicles in CBX-treated ovaries were at pre-antral stages. Meanwhile, not only was the number of follicles in the CBXtreated group apparently lower than that in the control, but also the size of ovary tissue was $85.7 \%$ smaller than that in the control (Fig. 3).

Considering that the expression pattern of many gap junction genes in the ovary closely relates to fetal mouse development, especially the time point of parturition (Fig. 1), it is worthwhile to determine whether gap junction functions during this period and later determine the formation of primordial follicles. To address this issue, we designed a temporal gap junction blockade experiment.

In the first treatment group (19.5 dpc- + group), ovaries at $17.5 \mathrm{dpc}$ were cultured in a normal medium for 2 days, then equivalent to $19.5 \mathrm{dpc}$ in vivo, and CBX was added at a final concentration of $10 \mu \mathrm{M}$. After 5 days of culture with CBX, equal to $5 \mathrm{dpp}$ in vivo, cysts could be clearly identified in these ovaries (Fig. 4B). Oocyte number in cysts was 7.7-fold higher than that in the control group (963.2 \pm 25.3 vs $125.6 \pm 18.8$ oocytes). Meanwhile, primordial follicle number in $19.5 \mathrm{dpc}-+$ group ovaries was 1.7-fold lower than that in the control group (1803.7 \pm 95.3 vs $3033.2 \pm 45.9$ follicles) $(P<0.05)$ (Fig. 4C).

In the second group ( $1 \mathrm{dpp}-+$ group), ovaries at $17.5 \mathrm{dpc}$ were cultured for 3 days in a blocker-free medium (equal to $1 \mathrm{dpp}$ in vivo) and then CBX was added. After 4 days of culture, equal to $5 \mathrm{dpp}$ in vivo, the

Figure 4 Temporal effect of gap junction blockade on follicle assembly. (A) Diagram of the experiment design. Time course occupied by the red block indicates that the ovaries were treated with CBX within it. (B) IHC examination of $17.5 \mathrm{dpc}$ ovaries under different treatments after 7 days of culture. The left column presents an overall view of cultured tissues in the control (Ctrl), blocked at $19.5 \mathrm{dpc}(19.5 \mathrm{dpc}-+)$, blocked at $1 \mathrm{dpp}(1 \mathrm{dpp}-+)$, withdrawn at $19.5 \mathrm{dpc}(19.5 \mathrm{dpc}+-)$ and withdrawn at $1 \mathrm{dpp}(1 \mathrm{dpp}+-)$ groups. The right column shows a detailed view of the same slice. DDX4 was immunostained (reddish brown) to label the oocytes. The structure of a typical cyst is encircled by the dotted lines; the arrow indicates typical primordial follicle. Scale bar: $50 \mu \mathrm{m}$. (C) Developmental stage statistics of oocytes. The letters indicate significant differences between groups $(P<0.05$; ANOVA). Data are presented as means \pm S.E.M. This experiment was repeated five times independently. 
numbers of both oocytes in cysts $(183.2 \pm 23.5$ vs $125.6 \pm 18.8$ oocytes) and primordial follicles $(2938.2 \pm 22.6$ vs $3033.2 \pm 45.9$ follicles) of this group were not significantly different from that in the control group (Fig. 4B and C).

In the third group (19.5 dpc +- group), ovaries at $17.5 \mathrm{dpc}$ were cultured in the CBX-containing medium for 2 days and then in the normal medium for the rest of the 5 days, which was the opposite of that in the first group. As shown by the histological results, primordial follicle number in this group (2597.6 \pm 31.9 follicles) was $14.4 \%$ lower than that in the control group and $11.6 \%$ lower than that in the $1 \mathrm{dpp}-+$ group $(P<0.05)$, but it was still 1.44-fold higher than that in the $19.5 \mathrm{dpc}-+$ group $(P<0.05)$ (Fig. 4B and C).

In the fourth group (1 dpp +- group), ovaries were treated with CBX 1 day longer than that in the $19.5 \mathrm{dpc}+-$ group. Interestingly, primordial follicle formation in this group (1900.3 \pm 36.2 follicles) was blocked, which was significantly different from the control group $(P<0.05$; Fig. 4B and $\mathrm{C})$.

We can conclude that the groups that were not blocked during the period of $19.5 \mathrm{dpc}$ to $1 \mathrm{dpp}$, including the $1 \mathrm{dpp}-+, 19.5 \mathrm{dpc}+-$ and control groups, not only formed primordial follicles in comparable number but also formed primary follicles. However, in the groups blocked between $19.5 \mathrm{dpc}$ and $1 \mathrm{dpp}$, there were hardly any primary follicles found (Fig. 4B and C). Furthermore, all treatments did not significantly affect the number of total oocytes.

\section{Specific inhibition of Gja1 and Gja4 did not affect primordial follicle formation}

Gja1 and Gja4 are the two major gap junction genes in the mature ovary. To determine their role during primordial follicle formation, we used siRNA technology to knock down their expression perinatally and then evaluated the histological changes within the ovary. We confirmed that siRNAs targeting either Gja1 or Gja4 work effectively and specifically and that a cocktail of these two siRNAs also inhibited both Gja1 and Gja4 expression (Fig. 5B). However, histological examination did not show apparent differences between the individual treatment groups, except the cocktailtreated group (Fig. 5A). In detail, non-target control ovaries developed primordial follicles similar in both number and morphology. Meanwhile, primordial follicle formation was not affected in either Si-Gja1 or Si-Gja4 ovaries. However with simultaneous mRNA interference, cyst breakdown was slightly inhibited (Fig. 5A). The oocyte number was threefold higher in the cysts of the Si-Gja1/Gja4 group than that in the control group ( $363.2 \pm 55.2$ vs $120.2 \pm 19.8$ oocytes, $P<0.05$; Fig. 5C). However, the primordial follicle number or the total oocyte number did not significantly differ between the groups.
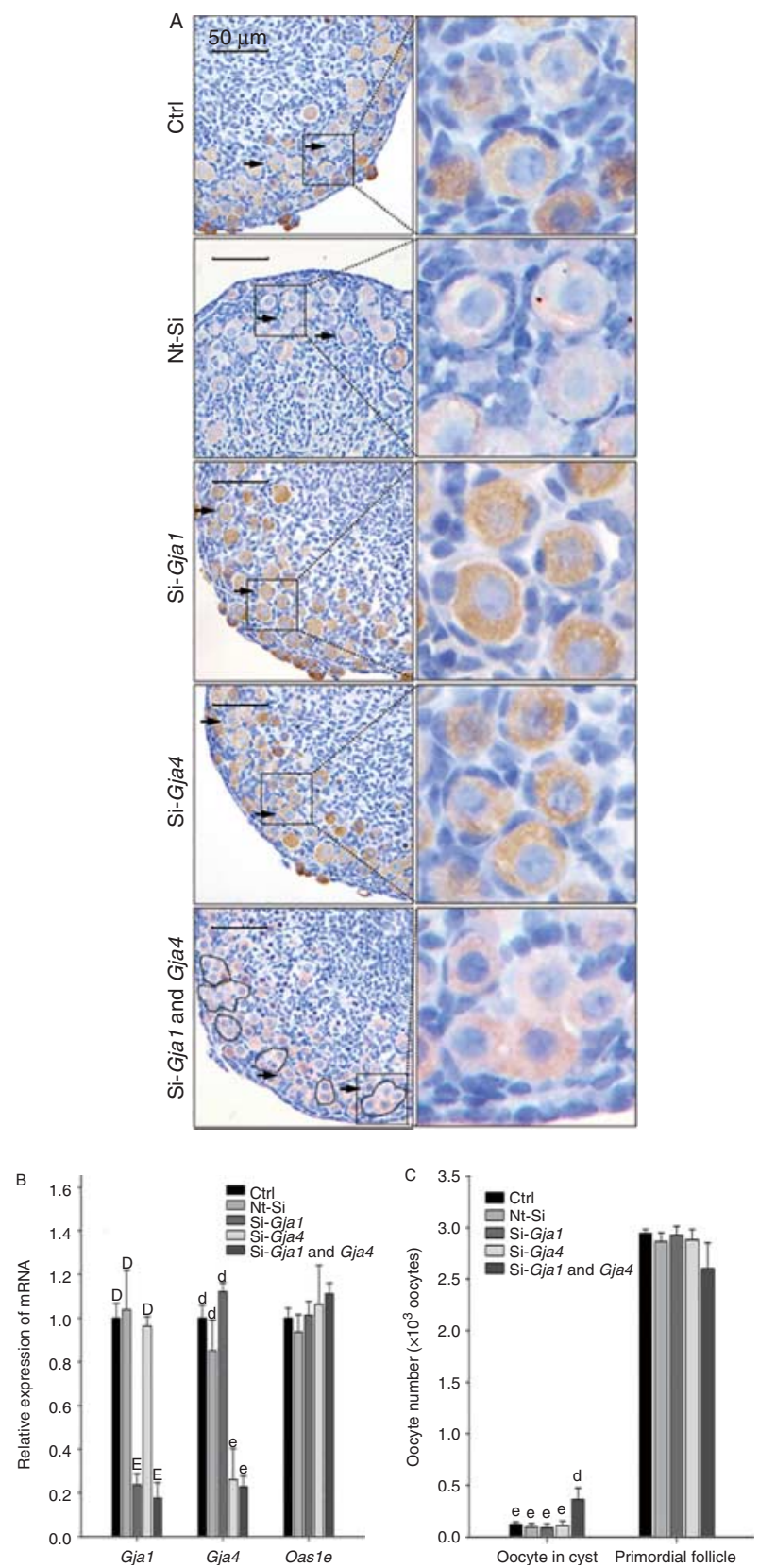

Figure 5 Role of Gja 1 and Gja4 in follicle assembly in in vitro cultured fetal ovaries. (A) Histological analysis of $17.5 \mathrm{dpc}$ fetal ovaries treated with Gja1-specific and/or Gja4-specific siRNA after 7 days of culture. The left column presents an overall view of cultured tissues in the control (Ctrl), non-target siRNA (Nt-Si), Gja1-specific siRNA (Si-Gja1), Gja4-specific siRNA (Si-Gja4) and cocktail siRNA (Si-Gja1 and Si-Gja4) groups. The right column shows a detailed view of the same slice. DDX4 was immunostained (reddish brown) to label the oocytes. The structure of a typical cyst is encircled by the dotted lines; the arrow indicates typical primordial follicle. Scale bar: $50 \mu \mathrm{m}$. (B) The knockdown efficiency of siRNA targeting Gja1 and Gja4. The expression of Gja1 and Gja4 in each treatment group was evaluated by qRT-PCR. The letters indicate significant differences between groups $(P<0.05$; ANOVA). All values were normalized to Gapdh. (C) Developmental stage statistics of oocytes. The letters indicate significant differences between groups $(P<0.05 ;$ ANOVA). Data are presented as means \pm S.E.M. All the experiments were repeated five times independently. 

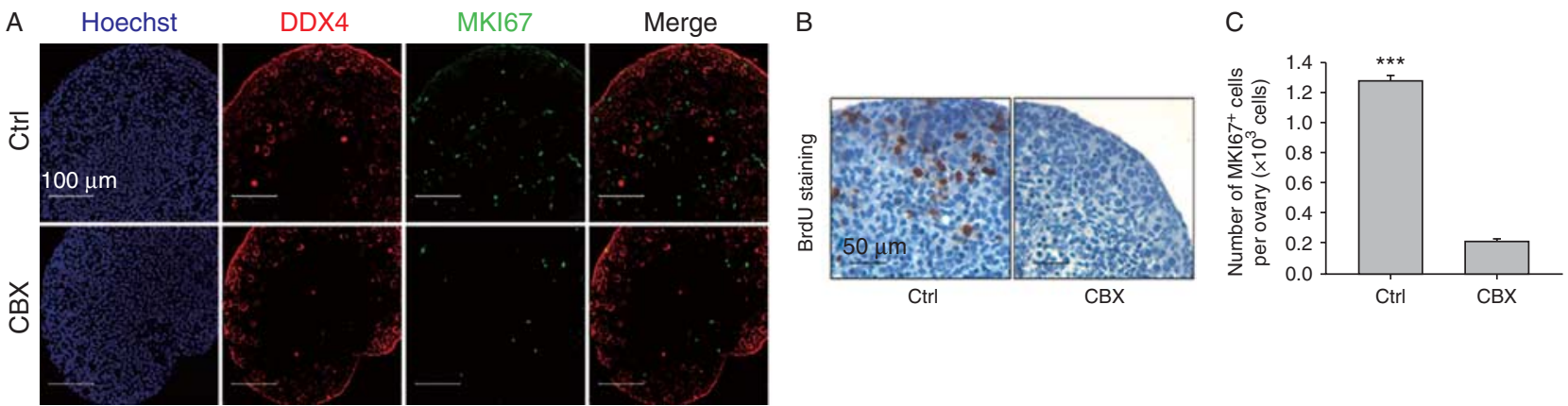

Figure 6 Effect of gap junction blockade on ovarian cell proliferation. (A) IF examination of MKI67 expression affected by CBX treatment in 17.5 dpc fetal ovaries after 3 days of culture. The MKI67 protein (in green) expression patterns in the ovaries of the control (Ctrl) and CBXCBX-treated groups are shown, in which ovarian cell nuclei appear in blue and the oocyte-specific protein DDX4 is displayed in red. Hoechst signal in each sample was exposed for $50 \mathrm{~ms}$, DDX4 for $200 \mathrm{~ms}$ and MKI67 for $200 \mathrm{~ms}$. Scale bar: $100 \mu \mathrm{m}$. (B) IHC examination of BrdU incorporation affected by CBX treatment in $17.5 \mathrm{dpc}$ fetal ovaries after 3 days of culture. BrdU was immunostained (reddish brown) to label the proliferating cells. Scale bar: $50 \mu \mathrm{m}$. (C) Statistical analysis of MKI67-positive cells. The asterisk indicates significant differences between groups $(P<0.001 ; t$-test). Data are presented as means \pm s.E.M. All the experiments were repeated three times independently.

\section{Gap junction blockade inhibited ovarian somatic cell proliferation}

We used MKI67 as a proliferation marker to investigate the cellular conditions under the gap junction blocker treatment. After 3 days of culture from $17.5 \mathrm{dpc}$, equal to $1 \mathrm{dpp}, \mathrm{CBX}$ treatment led to a significant difference in proliferation in the treated ovaries compared with the control group. The control ovaries showed many actively dividing and MKI67-positive cells, especially among the ovarian cords and surrounding oocytes (Fig. 6A). However, with GJC blockade, the number of MKI67positive somatic cells declined significantly (1276.4 \pm 33.2 vs $216.2 \pm 14.2$ OSCs, $P<0.05$; Fig. 6C). Similarly, the $\mathrm{BrdU}$ incorporation assay showed the disappearance of BrdU-positive cells due to the CBX treatment (Fig. 6B).

\section{Relationship between gap junction communication and signaling that is essential for follicle assembly}

To explore the relationship between GJC and proven key factors involved in follicle assembly, we subjected a 3-day cultured $17.5 \mathrm{dpc}$ ovary to qRT-PCR assays to examine the changes in the expression of these genes. First, we tested the essential genes for primordial follicle formation, meiosis or OSC development, including folliculogenesis-specific basic helix-loop-helix (Figla), Y box protein 2 ( $Y b x 2$, also Msy2), NOBOX oogenesis homeobox (Nobox), spermatogenesis- and oogenesisspecific basic helix-loop-helix 1 (Soh/h1), Foxl2 and Iroquois-related homeobox 3 (Irx3). Interestingly, almost all oocyte-specific genes were not affected. Figla was the only oocyte-expressing gene that changed significantly, but it increased only 1.2-fold compared with the control group $(P<0.05$; Fig. $7 \mathrm{~A})$. The expression of both OSCspecific genes tested decreased due to the CBX treatment. Particularly, Foxl 2 decreased by $45.4 \%$ and Irx3 decreased by 29.9\% ( $P<0.05$; Fig. 7A). Second, considering that gap junction blockade with $\mathrm{CBX}$ results in a similar phenotype to that with DAPT $(N-[N-(3,5-$ difluorophenacetyl-L-alanyl)]-S-phenylglycine $t$-butyl ester), a Notch signaling inhibitor (Trombly et al. 2009, Guo et al. 2012), we investigated the relationship between GJC and Notch pathways by examining the alteration of the expression of related genes after either CBX or DAPT treatment. We determined the expression of ten essential Notch pathway members under CBX treatment. After 3 days of culture in the gap junction blockade medium, most Notch pathway genes were stable, except notch 2 (Notch2), transducin-like enhancer of split 1, homolog of Drosophila E (spl) (Tle1) and Tle6. Specifically, Notch 2 was downregulated by $64.4 \%$ and Tle 6 was downregulated by $39.9 \%$, while Tle 1 was upregulated by $57.7 \%(P<0.05$; Fig. 7B).

We then examined the fetal ovary-expressing gap junction genes under DAPT treatment. Interestingly, seven out of eight affected genes were upregulated, including Gja1 (increased by 1.6-fold), Gja4 (1.3-fold), Gja8 (1.7-fold), Gjb4 (1.9-fold), Gjc1 (1.2-fold), Gjc3 (3.6-fold) and Gjd4 (4.1-fold) $(P<0.05)$. Furthermore, Gjc2 was the only downregulated gene, decreased by $66 \%(P<0.05$; Fig. 7C).

\section{Discussion}

In this study, the physiological action of GJC was proved to be important for primordial follicle formation. First, in the perinatal mouse ovary, multiple gap junction genes were upregulated and the gap junction structure was established. Second, inhibition of gap junctions during fetal age not only prevented primordial follicle assembly in vitro, but also resulted in the failure of antral follicle development in the kidney transplant culture. In addition, GJC in the ovary, from $19.5 \mathrm{dpc}$ to $1 \mathrm{dpp}$, was essential for primordial follicle assembly. We further proved that multiple gap junction genes may participate in primordial follicle assembly and that Notch signaling 

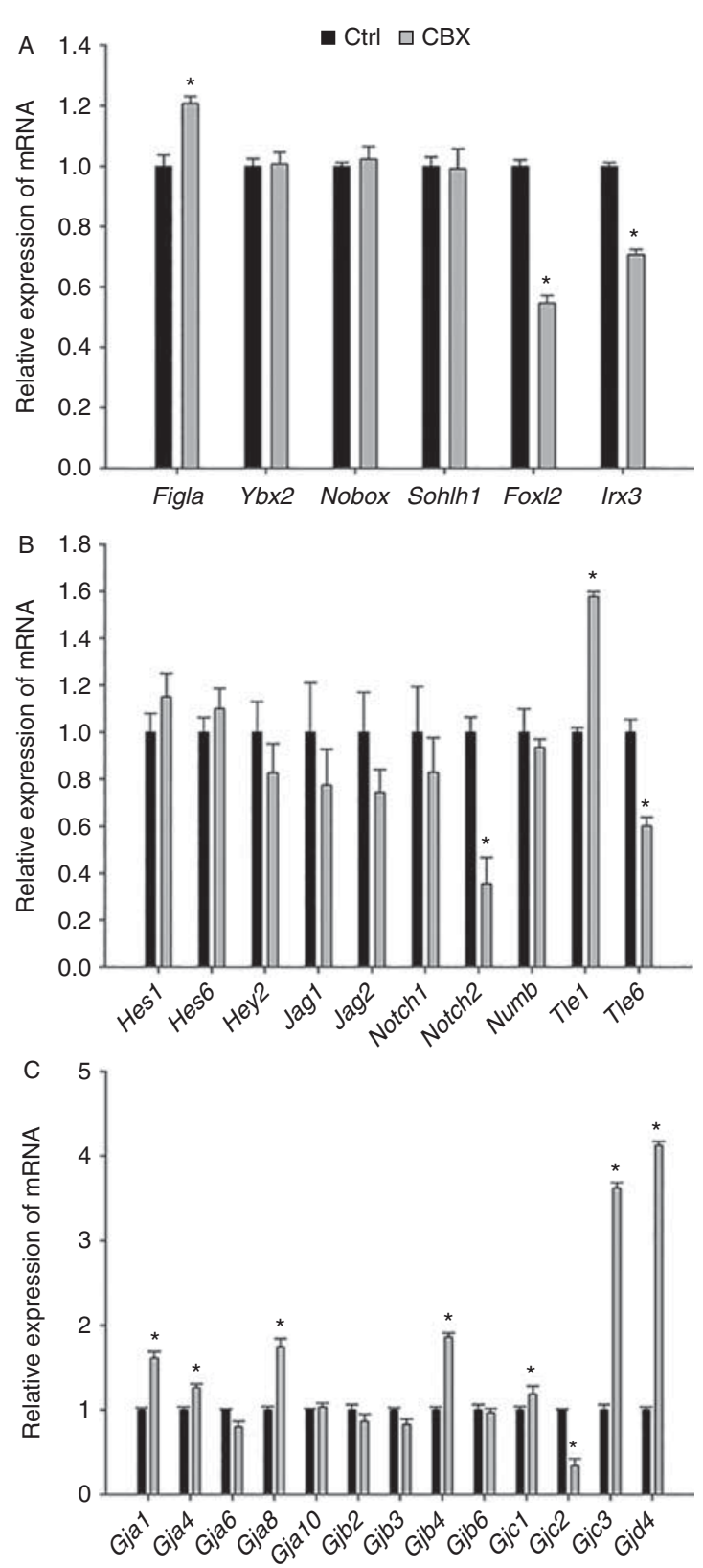

Figure 7 Interaction between gap junction communication and follicle assembly-related factors. (A) Effect of CBX treatment on the mRNA expression of essential follicle assembly genes in $17.5 \mathrm{dpc}$ fetal ovaries after 3 days of treatment. The relative expression of these genes was evaluated by qRT-PCR. All values were normalized to Gapdh. The asterisk indicates significant differences between the control (Ctrl) and CBX-treated groups $(P<0.05$; $t$-test). (B) Effect of CBX treatment on the mRNA expression of Notch pathway members in $17.5 \mathrm{dpc}$ fetal ovaries after 3 days of treatment. The relative expression of these genes was evaluated by qRT-PCR. All values were normalized to Gapdh. The asterisk indicates significant differences between the Ctrl and CBXtreated groups $(P<0.05 ; t$-test). (C) Effect of DAPT treatment on the mRNA expression of 13 perinatal ovary-expressing gap junction genes in $17.5 \mathrm{dpc}$ fetal ovaries after 3 days of treatment. The relative expression of these genes was evaluated by qRT-PCR. All values were normalized to Gapdh. The asterisk indicates significant differences between the control $(\mathrm{Ctrl})$ and DAPT-treated groups $(P<0.05 ; t$-test). All the experiments were repeated three times independently. may be important for the establishment of GJC in the ovary and further determine the differentiation of OSCs.

The increase of gap junctions in the ovary near birth implies that ovarian cellular communication may occur at this time. The issue is consistent with another ultramicroscopic study, where the original appearance of gap junction structure was observed at $17.5 \mathrm{dpc}$ and the structures increased along with fetal mouse ovary development (Mitchell \& Burghardt 1986). As has been proved by this study, the gap junction genes upregulated at this time may participate in the construction of the structure. Specifically, two downregulated gap junction genes may work earlier than the perinatal period, within which time abundant gap junction structures have also been found among OSCs (Mitchell \& Burghardt 1986). Yet, an unsolved question about the existence of gap junctions in the fetal ovary is how to explain the distributions as well as functions of these confirmed gap junction genes. The main problem in getting convincing answers is that there are too many gap junction genes waiting for evaluation at this time, and therefore, greater efforts are needed before we can get a clearer understanding of GJC in the fetal ovary.

A possible reason for neglecting the role of gap junctions during early folliculogenesis may be that there are few related phenotypes in gap junction gene knockout animals. The deficiency of either Gja1 or Gja4, the two most essential gap junction genes for follicle growth (Fagbohun \& Downs 1991, Downs 1995), does not affect primordial follicle formation (Simon et al. 1997, Juneja et al. 1999). One explanation for this finding may be the changes in the rest of the gap junction family members when a particular one is absent (Juneja 2003). Our blockade results provided the evidence of gap junction involvement in follicle assembly, but the gene-specific knock-down results remind us that the detailed functions of the genes are far from being well resolved. In this study, since the double knock-down effect on follicle assembly was not comparable to chemical blockade results, it implies that a core regulation mechanism is yet to be discovered. Furthermore, considering that substance exchange through gap junctions is gene-specific (Ek-Vitorin \& Burt 2013), gap junction-conducted cellular communication between different types of ovarian cells is probably a complex transporting network involving various regulatory factors (Nicholson et al. 2000). Thus, we believe that simply interpreting the results of chemical blockade, as well as Gja1/Gja4 knock-down result, as an additive effect to certain singular substances is not enough; with the growing knowledge of ovarian cell classification, future investigations in fetal ovarian gap junctions will acquire more details about which gap junction gene is expressed in which cell type (Rastetter et al. 2014, Zheng et al. 2014).

Postnatal proliferation of OSCs has been observed in many studies, but their physiological role is still unclear. 
Here, OSC proliferation in 3-day in vitro cultured $17.5 \mathrm{dpc}$ (equal to $1 \mathrm{dpp}$ ) ovaries was significantly inhibited by the gap junction blockade. This result for the first time linked OSC postnatal proliferation to primordial follicle assembly. Meanwhile, our unpublished data revealed that not only the synchronized developmental stage of oocytes and OSCs, but also the matched cell number, among them, are essential for primordial follicle assembly. Hence, whether the postnatal proliferation of OSCs determines the proper OSC number, which is necessary for the establishment of the primordial follicle pool, is a question that remains to be answered.

When examining the impact of gap junction blockade on genes that are essential for follicle assembly, we found that oocyte-specific expressed genes, such as Figla, Nobox, and Sohlh1, are almost not affected (Soyal et al. 2000, Rajkovic et al. 2004, Pangas et al. 2006). Meanwhile, we found that gap junction blockade did not disturb ongoing oocyte meiosis (Wang et al. 2015). This indicates that perinatal oocyte development may not strongly depend on GJC. On the contrary, all tested OSCexpressing genes, such as Fox/2, Iroquois-related homeobox 3 (Irx3) and Notch2, were downregulated by the blockade (Jorgensen \& Gao 2005, Xu \& Gridley 2013, Georges et al. 2014). It is worthwhile to explore the relationship of GJC with the Notch signaling pathway in the future because Notch has been proved to be important for primordial follicle assembly (Trombly et al. 2009, Guo et al. 2012, Xu \& Gridley 2013). Together, it implies that GJC may be more important for OSC differentiation rather than for oocyte development. Interestingly, in a germ cell-deleted model where the oocyte-OSC communication is disrupted but the OSCOSC communication is intact, the establishment of the female gonad development pathway is not effected. This supports the suggestion that the somatic cell population is not affected by the oocyte-OSC GJC (Maatouk et al. 2012). Taken together, the development of OSCs may be the target of gap junctions, and hence, the origin of upstream control signals could be an interesting topic in the future.

A big question is which gap junction member is the core junction during follicle assembly. Although we have tried to explore the function of certain gap junction genes, such as Gja1 and Gja4, the result is obviously not a final answer to that question. The main problem, as mentioned above, is that there are too many gap junction genes expressed within the fetal ovary. It would be demanding work to check the function of all these genes one by one. However, we can still adopt some proven follicle-assembly-failure model to examine whether the related signaling pathway affects certain gap junctions, such as the downregulated Gjc2 in DAPT-treated ovaries.

In conclusion, gap junctions appearing in the fetal ovary near birth play a role in the regulation of primordial follicle formation and may have cross-talking to Notch signalings. Gap junction proteins, along with related processes, may provide greater understanding about the cell communication network during primordial follicle assembly. However, the exact regulation of gap junction function in primordial follicle formation still needs further study.

\section{Supplementary data}

This is linked to the online version of the paper at http://dx.doi. org/10.1530/REP-15-0282.

\section{Declaration of interest}

The authors declare that there is no conflict of interest that could be perceived as prejudicing the impartiality of the research reported.

\section{Funding}

This work was supported by the National Basic Research Program of China (2013CB945501; 2012CB944701) and Chinese Universities Scientific Fund (2015QC018). The funders had no role in the study design, collection, analysis and interpretation of the data, the writing of the report, or the decision to submit the article for publication.

\section{References}

Ackert CL, Gittens JE, O’Brien MJ, Eppig JJ \& Kidder GM 2001 Intercellular communication via connexin43 gap junctions is required for ovarian folliculogenesis in the mouse. Developmental Biology 233 258-270. (doi:10.1006/dbio.2001.0216)

Bukovsky A, Caudle MR, Svetlikova M, Wimalasena J, Ayala ME \& Dominguez R 2005 Oogenesis in adult mammals, including humans. Endocrine 26 301-316. (doi:10.1385/ENDO:26:3:301)

Burgoyne P \& Baker T 1981 Oocyte depletion in XO mice and their XX sibs from 12 to 200 days post partum. Journal of Reproduction and Fertility $\mathbf{6 1}$ 207-212. (doi:10.1530/jrf.0.0610207)

Davidson JS, Baumgarten IM \& Harley EH 1986 Reversible inhibition of intercellular junctional communication by glycyrrhetinic acid. Biochemical and Biophysical Research Communications 134 29-36. (doi:10.1016/0006-291X(86)90522-X)

Downs SM 1995 The influence of glucose, cumulus cells, and metabolic coupling on ATP levels and meiotic control in the isolated mouse oocyte. Developmental Biology 167 502-512. (doi:10.1006/ dbio.1995.1044)

Ek-Vitorin JF \& Burt JM 2013 Structural basis for the selective permeability of channels made of communicating junction proteins. Biochimica et Biophysica Acta 1828 51-68. (doi:10.1016/j.bbamem. 2012.02.003)

Fagbohun C \& Downs S 1991 Metabolic coupling and ligandstimulated meiotic maturation in the mouse oocyte-cumulus cell complex. Biology of Reproduction 45 851-859. (doi:10.1095/biolreprod45.6.851)

Georges A, Auguste A, Bessiere L, Vanet A, Todeschini AL \& Veitia RA 2014 FOXL2: a central transcription factor of the ovary. Journal of Molecular Endocrinology 52 R17-R33. (doi:10.1530/JME-13-0159)

Guo M, Zhang H, Bian F, Li G, Mu X, Wen J, Mao G, Teng Z, Xia G \& Zhang M 2012 P4 down-regulates Jagged2 and Notch1 expression during primordial folliculogenesis. Frontiers in Bioscience 4 2731-2744. (doi:10.2741/E579) 
Jorgensen JS \& Gao L 2005 Irx3 is differentially up-regulated in female gonads during sex determination. Gene Expression Patterns 5 756-762. (doi:10.1016/j.modgep.2005.04.011)

Juneja SC 2003 mRNA expression pattern of multiple members of connexin gene family in normal and abnormal fetal gonads in mouse. Indian Journal of Physiology and Pharmacology 47 147-156.

Juneja SC, Barr KJ, Enders GC \& Kidder GM 1999 Defects in the germ line and gonads of mice lacking Connexin43. Biology of Reproduction 60 1263-1270. (doi:10.1095/biolreprod60.5.1263)

Kidder G \& Mhawi A 2002 Gap junctions and ovarian folliculogenesis. Reproduction 123 613-620. (doi:10.1530/rep.0.1230613)

Krysko DV, Mussche S, Leybaert L \& D'Herde K 2004 Gap junctional communication and connexin43 expression in relation to apoptotic cell death and survival of granulosa cells. Journal of Histochemistry and Cytochemistry 52 1199-1207. (doi:10.1369/jhc.3A6227.2004)

Kumar NM \& Gilula NB 1996 The gap junction communication channel. Cell 84 381-388. (doi:10.1016/S0092-8674(00)81282-9)

Lei L, Zhang H, Jin S, Wang F, Fu M, Wang H \& Xia G 2006 Stage-specific germ-somatic cell interaction directs the primordial folliculogenesis in mouse fetal ovaries. Journal of Cellular Physiology 208 640-647. (doi:10. 1002/jcp.20702)

Li G, Zhang H, Wang Y, Wen J, Teng Z, Mao G, Wang J, Guo M, Mu X \& Xia G 2011 Stage-specific mice ovarian somatic cell is involved in primordial folliculogenesis. Frontiers in Bioscience 3 1025-1033. (doi:10.2741/e235)

Lo CW 1999 Genes, gene knockouts, and mutations in the analysis of gap junctions. Developmental Genetics 24 1-4. (doi:10.1002/(SICI)15206408(1999)24:1/2<1::AID-DVG1>3.0.CO;2-U)

Maatouk DM, Mork L, Hinson A, Kobayashi A, McMahon AP \& Capel B 2012 Germ cells are not required to establish the female pathway in mouse fetal gonads. PLOS ONE 7 e47238. (doi:10.1371/journal.pone. 0047238)

Mitchell PA \& Burghardt RC 1986 The ontogeny of nexuses (gap junctions) in the ovary of the fetal mouse. Anatomical Record 214 283-288. (doi:10.1002/ar.1092140307)

Mork L, Maatouk DM, McMahon JA, Guo JJ, Zhang P, McMahon AP \& Capel B 2012 Temporal differences in granulosa cell specification in the ovary reflect distinct follicle fates in mice. Biology of Reproduction $\mathbf{8 6}$ 37. (doi:10.1095/biolreprod.111.095208)

Nicholson BJ, Weber PA, Cao F, Chang H, Lampe P \& Goldberg G 2000 The molecular basis of selective permeability of connexins is complex and includes both size and charge. Brazilian Journal of Medical and Biological Research 33 369-378. (doi:10.1590/S0100-879X2000000400002)

Pangas SA, Choi Y, Ballow DJ, Zhao Y, Westphal H, Matzuk MM \& Rajkovic A 2006 Oogenesis requires germ cell-specific transcriptional regulators Sohlh1 and Lhx8. PNAS 103 8090-8095. (doi:10.1073/pnas. 0601083103)

Pepling ME \& Spradling AC 2001 Mouse ovarian germ cell cysts undergo programmed breakdown to form primordial follicles. Developmental Biology 234 339-351. (doi:10.1006/dbio.2001.0269)
Rajkovic A, Pangas SA, Ballow D, Suzumori N \& Matzuk MM 2004 NOBOX deficiency disrupts early folliculogenesis and oocyte-specific gene expression. Science 305 1157-1159. (doi:10.1126/science.1099755)

Rastetter RH, Bernard P, Palmer JS, Chassot AA, Chen H, Western PS, Ramsay RG, Chaboissier MC \& Wilhelm D 2014 Marker genes identify three somatic cell types in the fetal mouse ovary. Developmental Biology 394 242-252. (doi:10.1016/j.ydbio.2014.08.013)

Simon AM, Goodenough DA, Li E \& Paul DL 1997 Female infertility in mice lacking connexin 37. Nature 385 525-529. (doi:10.1038/385525a0)

Smith P, Wilhelm D \& Rodgers RJ 2014 Development of mammalian ovary. Journal of Endocrinology 221 R145-R161. (doi:10.1530/JOE-14-0062)

Soyal SM, Amleh A \& Dean J 2000 FIG $\alpha$, a germ cell-specific transcription factor required for ovarian follicle formation. Development 127 4645-4654.

Trombly DJ, Woodruff TK \& Mayo KE 2009 Suppression of Notch signaling in the neonatal mouse ovary decreases primordial follicle formation. Endocrinology 150 1014-1024. (doi:10.1210/en.2008-0213)

Valiunas V, Polosina YY, Miller H, Potapova IA, Valiuniene L, Doronin S, Mathias RT, Robinson RB, Rosen MR, Cohen IS et al. 2005 Connexinspecific cell-to-cell transfer of short interfering RNA by gap junctions. Journal of Physiology 568 459-468. (doi:10.1113/jphysiol.2005.090985)

Wang Y, Teng Z, Li G, Mu X, Wang Z, Feng L, Niu W, Huang K, Xiang X, Wang C et al. 2015 Cyclic AMP in oocytes controls meiotic prophase I and primordial folliculogenesis in the perinatal mouse ovary. Development 142 343-351. (doi:10.1242/dev.112755)

Wen J, Zhang H, Li G, Mao G, Chen X, Wang J, Guo M, Mu X, Ouyang H, Zhang $M$ et al. 2009 PAR6, a potential marker for the germ cells selected to form primordial follicles in mouse ovary. PLoS ONE 4 e7372. (doi:10. 1371/journal.pone.0007372)

Wolvetang EJ, Pera MF \& Zuckerman KS 2007 Gap junction mediated transport of shRNA between human embryonic stem cells. Biochemical and Biophysical Research Communicatons 363 610-615. (doi:10.1016/ j.bbrc.2007.09.035)

Xu J \& Gridley T 2013 Notch2 is required in somatic cells for breakdown of ovarian germ-cell nests and formation of primordial follicles. BMC Biology 11 13. (doi:10.1186/1741-7007-11-13)

Zhang H, Zheng W, Shen Y, Adhikari D, Ueno H \& Liu K 2012 Experimental evidence showing that no mitotically active female germline progenitors exist in postnatal mouse ovaries. PNAS 109 12580-12585. (doi:10.1073/ pnas.1206600109)

Zheng W, Zhang H, Gorre N, Risal S, Shen Y \& Liu K 2014 Two classes of ovarian primordial follicles exhibit distinct developmental dynamics and physiological functions. Human Molecular Genetics 23 920-928. (doi:10.1093/hmg/ddt486)

Received 23 June 2015

First decision 16 July 2015

Revised manuscript received 2 November 2015

Accepted 9 November 2015 